\title{
Prevalência de aconselhamento para atividade física na Atenção Básica à Saúde: uma revisão sistemática
}

\section{Prevalence of physical activity counseling in Primary Health Care: a systematic review}

\section{AUTORES \\ Sheylane de Queiroz Moraes ${ }^{1}$ (ic Juliana Hegeto de Souza ${ }^{1}$ (iD) \\ Pablo Antônio Bertasso de Araújo ${ }^{1}$ (it) \\ Cassiano Ricardo Rech ${ }^{1}$ (D) \\ 1 Universidade Federal de Santa Catarina, Centro de Desportos. Florianópolis, Santa Catarina, Brasil. \\ CONTATO \\ Sheylane de Queiroz Moraes \\ moraessheylane@gmail.com \\ Campus Universitário Reitor João David \\ Ferreria Lima. Prédio Administrativo, Centro de Desportos, sala 200 - 2 andar, Trindade, Florianópolis, Santa Catarina, Brasil. CEP: 88040-900. \\ $\mathrm{DOI}$}

$10.12820 /$ rbafs. $24 \mathrm{e} 0073$

\begin{abstract}
RESUMO
O aconselhamento tem se mostrado promissor para mudança de comportamento. Este estudo teve como objetivo realizar uma revisão sistemática sobre a prevalência de aconselhamento para a prática de atividade física oferecido pelos profissionais de saúde e recebido pelos usuários no contexto da Atenção Básica à Saúde. Foram revisadas as bases de periódicos PsycINFO, EBSCOhost, PubMed, ScienceDirect, Web of Science, LILACS e SciELO. O risco de viés foi avaliado em todos os artigos. Foram incluídos na revisão 44 artigos, destes, 21 investigaram aconselhamento fornecido por profissionais de saúde e 23 analisaram aconselhamento recebido por usuários. A prevalência de aconselhamento variou de $11,8 \%$ a $95,0 \%$ entre os profissionais $(60,2 \pm 22,7 \%)$ e $4,5 \%$ a $61,6 \%$ entre usuários $(36,6 \pm 14,2 \%)$. Médicos são os profissionais mais investigados. Usuários com fatores de risco para as doenças crônicas são os que mais relatam receber aconselhamento. $\mathrm{O}$ aconselhamento ainda é incipiente na Atenção Básica à Saúde, especialmente pela baixa percepção de aconselhamento recebido pelos usuários. Existe a necessidade de padronizar métodos para avaliar o aconselhamento para prática de atividade física, especialmente no tempo, no tipo e na forma de aconselhar.
\end{abstract}

Palavras-chave: Aconselhamento; Atividade motora; Atenção primária à saúde; Promoção da saúde. ABSTRACT

Counseling has shown promise for behavior change. This study aims to carry out a systematic review on the prevalence of counseling for the practice of physical activity offered by health professionals and received by users in the context of Primary Health Care. We reviewed the journal bases PsycINFO, EBSCOhost, PubMed, ScienceDirect, Web of Science, LILACS and SciELO. The risk of bias was evaluated in all articles. In total, 44 articles were included, of which 21 investigated advice provided by health professionals and 23 analyzed advice received by users. The prevalence of counseling ranged from $11.8 \%$ to $95.0 \%$ among professionals (60.2 $\pm 22.7 \%)$ and $4.5 \%$ to $61.6 \%$ among users (36.6 $\pm 14.2 \%)$. Doctors are the most researched professionals. Users with risk factors for chronic diseases are the most reported to receive counseling. Counseling is still incipient in Primary Health Care, especially because of the low perception of counseling received by users. There is a need to standardize methods to evaluate physical activity counseling, especially in the kind and in the form of advice.

Keywords: Counseling; Motor activity; Primary health care; Health promotion.

\section{(cc) BY-NC-SA}

Este obra está licenciado com uma Licença

Creative Commons Atribuição-NãoComercial-

CompartilhaIgual 4.0 Internacional.

\section{Introdução}

Apesar das evidências dos benefícios da prática regular de atividade física (AF), a prevalência de inatividade física representa uma pandemia mundial ${ }^{1-5}$. Neste sentido, ações em diversos níveis têm sido implementadas para promoção de $\mathrm{AF}^{6}$. Uma estratégia que tem sido considerada promissora para mudança de comportamento é o aconselhamento ${ }^{7-10}$. Essa prática tem sido definida como uma forma de atuação do profissional que envolve a escuta, compreensão das pessoas e suporte para que elas planejem e tomem decisões mais favoráveis aos comportamentos saudáveis, incluindo a prática de $\mathrm{AF}^{11}$.

O aconselhamento para AF é uma importante estratégia para promover estilos de vida ativo em nível populacional ${ }^{8,10,12}$, principalmente quando realizado no contexto da Atenção Básica à Saúde (ABS), visto a elevada 
abrangência e capilaridade deste serviço ${ }^{13-16}$. Embora órgãos internacionais e nacionais recomendem a utilização do aconselhamento para $\mathrm{AF}$ na $\mathrm{ABS}^{13,17-19}$, não foi encontrado na literatura estudos que sumarizaram a prevalência de aconselhamento para AF neste contexto.

Revisões sistemáticas relacionadas ao tema abordaram os efeitos de intervenções ${ }^{8,20,21}$, as percepções dos profissionais a respeito de barreiras e facilitadores para aconselhar ${ }^{22}$ e a presença de aconselhamento em componentes curriculares na formação médica ${ }^{23}$. Assim, é importante identificar qual a frequência em que o aconselhamento para $\mathrm{AF}$ é realizado na $\mathrm{ABS}$, bem como os usuários e profissionais envolvidos, a fim de identificar o quanto essa prática está inserida no cotidiano dos serviços de saúde. Desse modo, o objetivo deste estudo foi realizar uma revisão sistemática da literatura sobre a prevalência de aconselhamento para a prática de $\mathrm{AF}$ oferecido pelos profissionais de saúde e recebido pelos usuários na $\mathrm{ABS}$.

\section{Métodos}

Esta revisão sistemática seguiu as diretrizes do guia Preferred Reporting Items for Systematic Reviews and Meta-analysis (PRISMA) ${ }^{24}$ e teve seu protocolo registrado e aprovado no International Prospective Register of Systematic Reviews (PROSPERO) sob o código CRD42017062179, o qual pode ser acessado pelo link https://www.crd.york.ac.uk/prospero.

Foram incluídos estudos observacionais com delineamento transversal, com amostra de adultos e idosos (faixa etária $\geq 18$ anos), realizados no contexto da $\mathrm{ABS}$ e que apresentavam como um dos componentes do aconselhamento a atividade física. Os artigos selecionados deveriam estar publicados na língua espanhola, inglesa ou portuguesa, disponíveis na íntegra e sem restrição de data.

Os seguintes critérios de exclusão foram aplicados: 1) estudos do tipo experimental; 2) livros e capítulos de livros, teses, dissertações, artigos de revisão, pontos de vista, ensaios e editoriais; 3) estudos não realizados no contexto da $\mathrm{ABS}$; 4) estudos que não utilizaram um dos componentes do aconselhamento para a atividade física; 5) estudos realizados com estudantes ou integrantes de programas de residência em saúde.

A busca sistemática foi realizada nas seguintes bases de periódicos: American Psychological Association (APA PsycINFO); EBSCOhost; PubMed; ScienceDirect; Web of Science; Literatura Latino-Americana e do Caribe em Ciências da Saúde (LILACS) e Scientific
Eletronic Library Online (SciELO). Além disso, uma pesquisa adicional foi realizada nas referências dos artigos selecionados.

A estratégia de busca eletrônica foi adaptada de acordo com as bases e utilizou descritores relacionados à atividade física, ao aconselhamento e à saúde; combinados por meio dos operadores booleanos "AND" e "OR". Na combinação dos descritores foi aplicada a sintaxe: ("exercise" OR "physical activity" OR "motor activity") AND ("counseling") AND ("primary health care" OR "public health").

A seleção dos estudos foi realizada em três etapas. A primeira etapa abrangeu a leitura dos títulos, a segunda dos resumos e a terceira, dos textos completos, realizadas de modo independente por dois revisores (JHS, SQM). Em caso de divergência entre os dois revisores, um terceiro revisor foi consultado (CRR). Para gerenciamento das referências e remoção dos duplicados foi utilizado software EndNote ${ }^{\circledR}$ X8, Thomson Reuters.

Um revisor (JHS) coletou as informações dos estudos selecionados e um segundo (SQM) revisou as informações coletadas. As controvérsias neste processo foram discutidas e decididas com a opinião do terceiro revisor (CRR). A extração de dados foi realizada em planilha eletrônica e organizadas os seguintes indicadores: 1) características do estudo (autor, ano de publicação, país do estudo); 2) características da população (sexo, grupo etário, tamanho da amostra); 3) características da exposição (profissionais responsáveis pelo aconselhamento e perfil dos usuários aconselhados); 4) prevalência de aconselhamento entre usuários e profissionais de saúde.

A análise do risco de viés dos artigos foi verificada pelos critérios publicados pelo The Joanna Briggs Institute $^{25}$. Dois revisores (SQM, PABA) classificaram os nove itens do instrumento de acordo com a escala proposta (sim, não, não claro ou não aplicável) das questões: o quadro da amostra foi apropriado para abordar a população alvo? Os participantes do estudo foram amostrados de maneira apropriada? $\mathrm{O}$ tamanho da amostra foi apropriado? Os sujeitos do estudo são descritos em detalhe? A análise dos dados foi realizada com cobertura suficiente da amostra identificada? Foram utilizados métodos válidos para identificação das variáveis? As variáveis foram medidas de maneira padronizada e confiável para todos os participantes? Houve análise estatística apropriada? A taxa de resposta foi adequada e, se não foi, a taxa de resposta foi gerenciada adequadamente? Quando necessário, um terceiro revisor (CRR) 


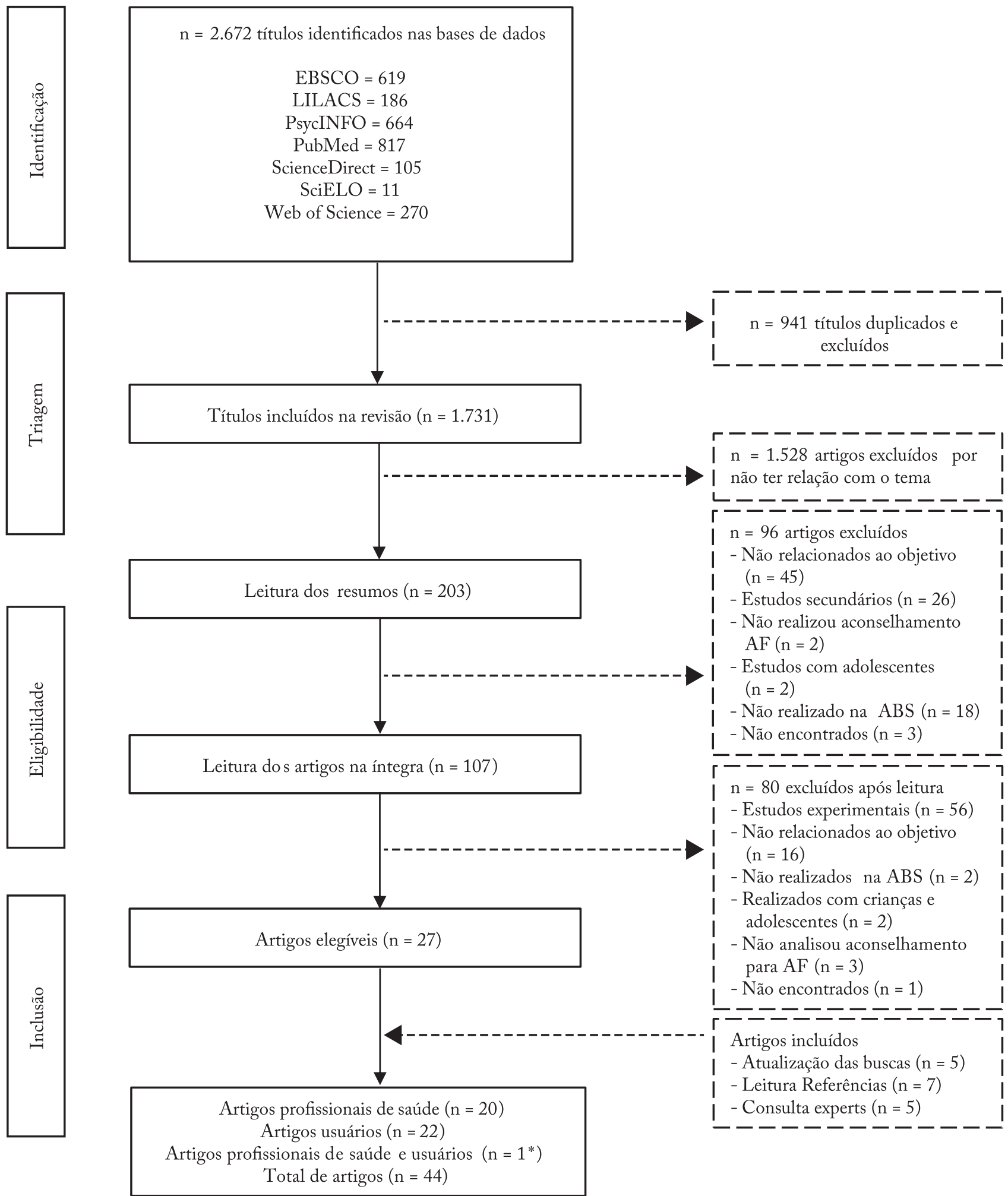

Figura 1 - Etapas de seleção dos artigos incluídos na revisão sistemática sobre a prevalência de aconselhamento para atividade física na Atenção Básica à Saúde.

* Artigo foi contabilizado nas duas categorias.

foi contatado para resolver quaisquer divergências. Para efeitos desta revisão sistemática, os artigos com mais de $70 \%$ de respostas "sim", foram classificados como baixo risco de viés; $50 \%$ a $70 \%$ de respostas "sim", como moderado risco de viés; e menos de 50\% de respostas "sim", como alto risco de viés ${ }^{26}$. 
A prevalência de aconselhamento para AF foi expressa por meio de porcentagem e apresentada em duas categorias: 1) aconselhamento oferecido pelos profissionais de saúde e; 2) aconselhamento para AF recebido por usuários da ABS.

\section{Resultados}

O processo de busca e aplicação dos critérios de inclusão/exclusão são apresentados na Figura 1. Ao final, foram incluídos 44 artigos publicados entre os anos de 1992 e 2018, destes, 21 (47,8\%) investigaram a prevalência de aconselhamento oferecido pelos profissionais da ABS e 23 artigos $(52,2 \%)$ avaliaram a prevalência de aconselhamento recebido pelos usuários, sendo que um destes artigos foi classificado em ambas as categorias ${ }^{27}$. A maior parte dos estudos foi desenvolvido nos Estados Unidos ( $n=14 ; 31,8 \%)$, seguido do Brasil ( $n=11$; $25,0 \%)$. Em relação à análise do risco de viés, a maioria dos artigos foi classificado com baixo risco de viés ( $\mathrm{n}=$ $26 ; 59,0 \%)$, seguido de moderado risco de viés $(\mathrm{n}=14$; $31,8 \%)$ e quatro foram $(9,0 \%)$ classificados com alto risco de viés (Tabela 1 e 2 ).

Dos artigos incluídos, 21 avaliaram a prevalência de aconselhamento para AF oferecido por profissionais da ABS (Tabela 1). A maior parte dos artigos reportou a realização de aconselhamento por médico $(n=10$; $47,6 \%)^{27-28,31,36-38,41,44-46}$ e por equipe multidisciplinar incluindo o médico $(\mathrm{n}=6 ; 28,6 \%)^{32-33,35,39,40,42}$; seguido por enfermeiro $(n=3 ; 14,3 \%)^{29,30,34}$ e por agente comunitário de saúde (também denominado em alguns países como visitante de saúde) $(\mathrm{n}=2 ; 9,5 \%)^{15,43}$. A prevalência de aconselhamento para AF referida pelos profissionais de saúde apresentou uma média de 60,2 $\pm 22,7 \%$ (Figura 2). Considerando apenas artigos com baixo risco de viés a média de aconselhamento foi de $63,7 \pm 26,9 \%$. Poucos artigos $(n=6 ; 28,6 \%)$ explicitaram a questão central utilizada para aferir a realização do aconselhamento para AF, e destes, dois apresentaram a mesma pergunta ${ }^{15,33}$.

Quinze artigos trataram exclusivamente do aconselhamento para a prática de $\mathrm{AF}$ e em cinco artigos esse aconselhamento foi tratado em conjunto a outras temáticas, como estilo de vida e alimentação saudável (Tabela 1). As práticas de aconselhamento mais abordadas nos artigos referem-se a característica dos profissionais que mais aconselham e barreiras percebidas pelos profissionais de saúde $(\mathrm{n}=10 ; 47,6 \%)$, seguidas, das características dos usuários mais aconselhados ( $\mathrm{n}=$ 8; 38,0\%) - Tabela 1 . O aconselhamento foi reportado por profissionais que de alguma maneira consideravam AF e estilo de vida como importante fator de saúde 28,31 e que eram familiarizados com AF, como os ativos fisicamente ${ }^{42,43}$, que conheciam as recomendações ${ }^{46}$ ou tinham cooperação com instituições esportivas ${ }^{28}$. A principal barreira para aconselhar foi a falta de tempo nos atendimentos ${ }^{29,30,32-34,37,40,42,44,46}$, e os usuários mais aconselhados pelos profissionais eram os que tinham fatores de risco à saúde ou doenças crônicas não transmissíveis $^{27,32,33-36,38,40,45}$ (Tabela 1).

Vinte e três artigos investigaram a prevalência de aconselhamento para AF recebido por usuários da ABS (Tabela 2). Destes, quinze $e^{12,47-50,52,54,55,58,59,61,64-67}$ trataram exclusivamente do aconselhamento para AF e cinco $^{27,51,56,60,63}$ incluíram aconselhamento para $\mathrm{AF}$ e alimentação saudável (Tabela 2). A maior parte dos artigos $(\mathrm{n}=13 ; 56,5 \%)$ utilizou uma pergunta central para mensurar o aconselhamento, questionava se nos últimos 12 meses o usuário havia recebido algum tipo de aconselhamento para AF (Tabela 2). Com base nesta pergunta central foi identificado que a maioria dos artigos reportou o médico como profissional responsável pelo aconselhamento $(n=13 ; 56,5 \%)^{27,47,53,55-}$ 57,59,61,63-67. A prevalência de aconselhamento para AF recebida pelos usuários variou de $4,5 \%$ a $61,6 \%$, com média de 36,6 \pm 14,2\% (Figura 2). Considerando apenas artigos com baixo risco de viés a média de aconselhamento foi de $35,0 \pm 15,2 \%$.

De modo geral, usuários com maior prevalência de aconselhamento foram aqueles com meia idade e idosos (de 40 a 75 anos) $)^{12,27,48-50,53-56,58,59,61,63,64,66}$, mulheres $^{12,48,50,53,57,58,64,67}$, pessoas com alguma doença crônica não transmissível e com excesso de peso corporal ou obesidade $12,27,47-50,52-54,56-61,63,64,66$.

\section{Discussão}

Os resultados da revisão sistemática indicaram que a prevalência de aconselhamento para AF referida pelos profissionais de saúde foi elevada, com média acima de $50 \%$, enquanto que a referida pelos usuários foi baixa, com média abaixo de $40 \%$. Os profissionais referem oferecer duas vezes mais aconselhamento do que o percebido pelos usuários da ABS. De modo geral, os médicos são os profissionais de saúde mais investigados; e os usuários que mais recebem aconselhamento para AF possuem fatores de risco e doenças crônicas não transmissíveis, como excesso de peso corporal e obesidade. Os estudos apresentaram em sua maior parte baixo risco de viés, porém as análises estratificadas por 
Tabela 1 - Síntese dos estudos realizados com profissionais de saúde a respeito do oferecimento de aconselhamento para atividade física na Atenção Básica à Saúde.

\begin{tabular}{|c|c|c|c|c|c|c|}
\hline Autor & País & $\mathrm{n}$ & $\begin{array}{l}\text { Profissional } \\
\text { entrevistado }\end{array}$ & Tema & Práticas de aconselhamento para AF referidas pelos profissionais & $\begin{array}{l}\text { Risco de } \\
\text { viés }\end{array}$ \\
\hline Bock et al. ${ }^{28}$ & GER & 260 & Médico & $\mathrm{AF}$ & $\begin{array}{l}\text { Profissional que + aconselha: } \\
\text { médicos das grandes cidades, que percebem sucesso em modificar AF } \\
\text { e com cooperação com clubes esportivos e de reabilitação cardíaca }\end{array}$ & Baixo \\
\hline Buchholz et al. ${ }^{29}$ & EUA & 96 & Enfermeiro & $\mathrm{AF}$ & $\begin{array}{l}\text { O que: } 30 \text { min de } \mathrm{AF} \text { todos dias da semana, caminhada e natação } \\
\text { Como: discute } \mathrm{AF} \text { e oferece materiais escritos } \\
\text { Barreiras: falta de tempo e receptividade do usuário, preocupações + } \\
\text { importantes a serem discutidas }\end{array}$ & Baixo \\
\hline Burns et al. ${ }^{30}$ & EUA & 396 & Enfermeiro & $\mathrm{AF}$ & $\begin{array}{l}\text { O que: caminhada, natação, ciclismo, atividades domésticas } \\
\text { Como: pergunta sobre AF, avalia altura e o peso } \\
\text { Barreiras: falta de tempo, outras preocupações + importantes }\end{array}$ & Baixo \\
\hline Cho et al. ${ }^{31}$ & KOR & 335 & Médico & ES & $\begin{array}{l}\text { Profissional que + aconselha: médicos que consideram ES um } \\
\text { importante fator de saúde }\end{array}$ & Moderado \\
\hline Douglas et al..$^{32}$ & $\mathrm{ESC}$ & 757 & $\begin{array}{l}\text { Médico, enfermeiro } \\
\text { e ACS }\end{array}$ & $\mathrm{AF}$ & $\begin{array}{l}\text { O que: caminhada } \\
\text { Profissional que + aconselha: enfermeiros e ACS } \\
\text { Usuário + aconselhado: excesso de peso } \\
\text { Barreiras: falta de tempo, de materiais educativos e de treinamento } \\
\text { específico }\end{array}$ & Moderado \\
\hline Florindo et al. ${ }^{33}$ & BRA & 529 & $\begin{array}{l}\text { Médico e } \\
\text { enfermeiro }\end{array}$ & $\mathrm{AF}$ & $\begin{array}{l}\text { Como: avalia AF utilizando questões gerais } \\
\text { Profissional que + aconselha: médicos } \\
\text { Barreiras: falta de tempo e de instalações para pacientes se envolverem } \\
\text { em AF }\end{array}$ & Baixo \\
\hline Florindo et al. ${ }^{15}$ & BRA & 269 & $\mathrm{ACS}$ & AF e AS & NR & Moderado \\
\hline $\begin{array}{l}\text { Grimstvedt et } \\
\text { al. }^{34}\end{array}$ & EUA & 318 & $\begin{array}{l}\text { Enfermeiro e } \\
{ }^{\mathrm{b}} \text { Assistente médico }\end{array}$ & $\mathrm{AF}$ & $\begin{array}{l}\text { O que: AF 3x na semana, recomendação de intensidade: "teste de } \\
\text { conversa" e caminhada moderada } \\
\text { Como: discute e pergunta sobre AF, avalia IMC e aparência física geral } \\
\text { Usuário +aconselhado: sedentários } \\
\text { Barreiras: falta de tempo, preocupações + importantes a serem discutidas }\end{array}$ & Alto \\
\hline Hidalgo et al. ${ }^{35}$ & BRA & 798 & $\begin{array}{l}\text { Médico, enfermeiro } \\
\text { e ACS }\end{array}$ & AF e ES & Usuário + aconselhado: $\uparrow I M C$, Dislipidemia, HAS, DM tipo 2 & Moderado \\
\hline Joyce et al. ${ }^{36}$ & IRL & 102 & Médico & $\mathrm{AF}$ & Usuário + aconselhado: obesidade e DM & Alto \\
\hline Kennedy et al..$^{37}$ & CAN & 330 & Médico & $\mathrm{AF}$ & $\begin{array}{l}\text { Barreiras: falta de tempo e educação continuada, ensino insuficiente } \\
\text { na graduação }\end{array}$ & Baixo \\
\hline${ }^{a}$ Kreuter et al. ${ }^{27}$ & EUA & 27 & Médico & $\mathrm{AF}$ e AS & Usuário + aconselhado: $\uparrow$ fatores de risco & Moderado \\
\hline $\begin{array}{l}\text { Morishita et } \\
\text { al. }^{38}\end{array}$ & JAP & 839 & Médico & $\mathrm{AF}$ & $\begin{array}{l}\text { Profissional que + aconselha: médicos entre } 30-40 \text { anos } \\
\text { Usuário + aconselhado: síndrome metabólica (DM e hiperlipidemia) }\end{array}$ & Moderado \\
\hline Morris et al. ${ }^{39}$ & CAN & 508 & $\begin{array}{l}\text { Clínico geral, } \\
\text { obstetra, parteira e } \\
\text { enfermeiro }\end{array}$ & $\mathrm{CP}$ & $\begin{array}{l}\text { Profissional que + aconselha: parteiras } \\
\text { Quando: estágios iniciais da gestação }\end{array}$ & Alto \\
\hline Omura et al. ${ }^{40}$ & EUA & 1251 & $\begin{array}{l}\text { Médico e } \\
\text { enfermeiro }\end{array}$ & $\mathrm{AF}$ & $\begin{array}{l}\text { Como: incentiva o aumento da } \mathrm{AF} \text {, avalia nível de } \mathrm{AF} \\
\text { Usuário + aconselhado: pacientes com + riscos } \\
\text { Barreiras: falta de tempo, descrença na adesão dos usuários }\end{array}$ & Baixo \\
\hline Petrella et al. ${ }^{41}$ & CAN & 13.166 & Médico & $\mathrm{AF}$ & $\begin{array}{l}\text { Como: aconselhamento verbal e pergunta sobre nível de AF } \\
\text { Profissional que }+ \text { aconselha: mulheres, } \geq 35 \text { anos, } \leq 6 \text { anos de prática, } \\
\text { de clínicas privadas e urbanas }\end{array}$ & Baixo \\
\hline Puig et al. ${ }^{42}$ & ESP & 245 & $\begin{array}{l}\text { Médico e } \\
\text { Enfermeiro }\end{array}$ & $\mathrm{AF}$ & $\begin{array}{l}\text { Profissional que + aconselha: enfermeiros e profissionais + ativos fisicamente } \\
\text { Barreiras: falta de tempo e treinamento, condições desfavoráveis de } \\
\text { trabalho }\end{array}$ & Baixo \\
\hline Santos et al. ${ }^{43}$ & BRA & 30 & $\mathrm{ACS}$ & $\mathrm{AF}$ & $\begin{array}{l}\text { Profissional que + aconselha: ACS com > média de passos diários de } \\
\text { caminhada }\end{array}$ & Baixo \\
\hline Sherman et al. ${ }^{44}$ & EUA & 422 & Médico & $\mathrm{AF}$ & Barreiras: falta de tempo & Moderado \\
\hline Smith et al. ${ }^{45}$ & EUA & 1211 & Médico & $\mathrm{AF}$ e AS & Usuário + aconselhado: com morbidades & Baixo \\
\hline Walsh et al. ${ }^{46}$ & EUA & 175 & Médico & $\mathrm{AF}$ & $\begin{array}{l}\text { O que: tipo, duração e frequência da AF Tempo: } 2-5 \text { minutos } \\
\text { Profissional que + aconselha: médicos de família, } \geq 35 \text { anos, }+ \\
\text { familiarizados com recomendações do ACSM } \\
\text { Barreiras: falta de tempo e de técnicas de aconselhamento, descrença } \\
\text { no efeito }\end{array}$ & Moderado \\
\hline
\end{tabular}

a Estudo realizado com usuários e profissionais, classificado em ambas as categorias; b Profissão semelhante ao enfermeiro, apenas com diferenças sutis. GER = Alemanha; EUA = Estados Unidos da América; KOR = Coreia; ESC = Escócia; BRA = Brasil; IRL = Irlanda; CAN = Canadá; JAP = Japão; ESP = Espanha; $\mathrm{n}$ = amostra ACS = agente comunitário de saúde/visitante de saúde; $\mathrm{AF}=$ atividade física; $\mathrm{ES}=$ estilo de vida; $\mathrm{AS}=$ alimentação saudável; $\mathrm{CP}=$ controle do peso; $\mathrm{NR}=$ não reportado; $\mathrm{IMC}=$ índice de massa corporal; HAS = hipertensão arterial sistêmica; $\mathrm{DM}=$ diabetes mellitus; $\mathrm{ACMS}=$ American College of Sports Medicine. 
Tabela 2 - Síntese dos estudos realizados com usuários a respeito do recebimento de aconselhamento para atividade física na Atenção Básica à Saúde.

\begin{tabular}{|c|c|c|c|c|c|c|c|c|}
\hline \multirow[t]{2}{*}{ Autor } & \multirow[t]{2}{*}{ País } & \multirow[t]{2}{*}{$\mathrm{n}$} & \multirow{2}{*}{$\begin{array}{l}\text { Idade } \\
\text { (anos) }\end{array}$} & \multirow[t]{2}{*}{ Tema } & \multicolumn{2}{|c|}{ Questão central da pesquisa } & \multirow{2}{*}{$\begin{array}{l}\text { Usuários que recebem aconselhamento } \\
\text { para } \mathrm{AF}\end{array}$} & \multirow[t]{2}{*}{ Risco de viés } \\
\hline & & & & & $\begin{array}{l}\text { Tempo de recordação } \\
\text { do aconselhamento }\end{array}$ & $\begin{array}{l}\text { Profissional } \\
\text { especificado }\end{array}$ & & \\
\hline Balde et al. ${ }^{47}$ & EUA & 146 & $\geq 65$ & $\mathrm{AF}$ & $\leq 12$ meses & Médico & Homens, casados, excesso de peso & Alto \\
\hline Barbosa et al. ${ }^{48}$ & $\mathrm{BR}$ & 1607 & $\geq 20$ & $\mathrm{AF}$ & Alguma vez & $\begin{array}{l}\text { Algum } \\
\text { profissional }\end{array}$ & $\begin{array}{l}\text { Mulheres, 20-59 anos, escolaridade formal, } \\
\text { HAS, OB }\end{array}$ & Baixo \\
\hline Croteau et al. ${ }^{49}$ & NZL & 8291 & $\geq 18$ & $\mathrm{AF}$ & $\leq 12$ meses & $\begin{array}{l}\text { Médico e } \\
\text { Enfermeiro }\end{array}$ & $\begin{array}{l}\text { Etnia maori, pacífica ou asiática, } 45 \text { - } 59 \\
\text { anos, excesso de peso ou OB, DCNT }\end{array}$ & Baixo \\
\hline Duro et al. ${ }^{50}$ & $\mathrm{BR}$ & 12.402 & $20-59$ & $\mathrm{AF}$ & $\leq 12$ meses & $\begin{array}{l}\text { Algum } \\
\text { profissional }\end{array}$ & $\begin{array}{l}\text { Mulheres, idades + avançadas, brancos, } \\
\text { casados, } \uparrow \text { renda, ativos fisicamente, ex- } \\
\text { fumantes, } \uparrow \text { IMC, DCNT }\end{array}$ & Baixo \\
\hline Flores et al. ${ }^{51}$ & $\mathrm{BR}$ & 1281 & $\geq 60$ & $\begin{array}{l}\mathrm{AF} \text { e } \\
\mathrm{AS}\end{array}$ & $\leq 12$ meses & $\begin{array}{l}{ }^{\mathrm{b}} \text { Algum } \\
\text { profissional }\end{array}$ & NR & Baixo \\
\hline Forjuoh et al. ${ }^{52}$ & EUA & 388 & $\geq 50$ & $\mathrm{AF}$ & $\leq 12$ meses & $\begin{array}{l}\text { Algum } \\
\text { profissional }\end{array}$ & $\begin{array}{l}\text { Autorrelato de saúde ruim, } \mathrm{OB} \text {, dificuldade } \\
\text { de andar } 1 / 4 \text { de milha, não realiza } 150 \mathrm{~min} \\
\text { de caminhada }\end{array}$ & Moderado \\
\hline Glasgow et al. ${ }^{53}$ & EUA & 1818 & $\geq 18$ & $\begin{array}{l}\mathrm{AF} \text { e } \\
\mathrm{ES}\end{array}$ & $\leq 12$ meses & Médico & $\begin{array}{l}\text { Mulheres, + velhos, não brancos, } \uparrow \mathrm{n}^{\circ} \\
\text { DCNT, > no consultas }\end{array}$ & Baixo \\
\hline Hafele et al. ${ }^{54}$ & $\mathrm{BR}$ & 525 & $\geq 18$ & $\mathrm{AF}$ & $\leq 12$ meses & NR & $\begin{array}{l}\text { Idades + avançadas, HAS, MCU, > n } \\
\text { consultas }\end{array}$ & Baixo \\
\hline Hallal et al. ${ }^{12}$ & $\mathrm{BR}$ & 972 & $20-69$ & $\mathrm{AF}$ & Alguma vez & $\begin{array}{l}{ }^{\mathrm{b}} \text { Algum } \\
\text { profissional }\end{array}$ & $\begin{array}{l}\text { Mulheres, } 50 \text { - } 59 \text { anos, } \uparrow \text { renda, autorrelato } \\
\text { de saúde ruim }\end{array}$ & Baixo \\
\hline Hinrichs et al. ${ }^{55}$ & GER & 1627 & $\geq 65$ & $\mathrm{AF}$ & $\leq 12$ meses & Médico & $\begin{array}{l}\text { Homens, < } 80 \text { anos, MCU (>5), sofrem } \\
\text { de dor }\end{array}$ & Moderado \\
\hline $\begin{array}{l}\text { Klumbiene et } \\
\text { al. }^{56}\end{array}$ & LIT & 2049 & $20-64$ & $\begin{array}{l}\mathrm{AF} \text { e } \\
\mathrm{AS}\end{array}$ & $\leq 12$ meses & Médico & $\begin{array}{l}50-64 \text { anos, } \uparrow \text { IMC, autorrelato de saúde } \\
\text { ruim, > no consultas }\end{array}$ & Baixo \\
\hline${ }^{a}$ Kreuter et al. ${ }^{27}$ & EUA & 680 & $\geq 18$ & $\begin{array}{l}\mathrm{AF} \text { e } \\
\mathrm{AS}\end{array}$ & $\leq 6$ meses & Médico & $\begin{array}{l}\text { Idades + avançadas, DCNT (DM, HAS, } \\
\text { dislipidemia), } \\
\uparrow \text { IMC }\end{array}$ & Moderado \\
\hline Ludt et al. ${ }^{57}$ & EUR & 3723 & $\geq 60$ & ES & $\leq 15$ meses & Médico & Mulheres e com maiores fatores de risco & Baixo \\
\hline $\begin{array}{l}\text { Martínez- } \\
\text { Gómez et al. }{ }^{58}\end{array}$ & ESP & 11951 & $\geq 18$ & $\begin{array}{l}\mathrm{AF} / \\
\mathrm{CAM}\end{array}$ & NR & $\begin{array}{l}\text { Médico e } \\
\text { Enfermeiro }\end{array}$ & $\begin{array}{l}\text { Mulheres, idade avançada, } \downarrow \text { nível } \\
\text { educacional, autorrelato de saúde ruim, } \downarrow \\
\text { AF global, } \uparrow \text { IMC }\end{array}$ & Baixo \\
\hline Pechter et al. ${ }^{59}$ & $\mathrm{EST}$ & 239 & $18-75$ & $\mathrm{AF}$ & NR & Médico & 50 - 75 anos, DCNT, $\uparrow$ IMC & Moderado \\
\hline Santos et al. ${ }^{60}$ & $\mathrm{BR}$ & 499 & $\geq 18$ & $\begin{array}{l}\text { AF e } \\
\text { AS }\end{array}$ & NR & $\begin{array}{l}{ }^{\mathrm{b}} \text { Algum } \\
\text { profissional }\end{array}$ & $>\mathrm{n}^{\circ}$ de morbidades (DCNT) & Moderado \\
\hline Shuval et al. ${ }^{61}$ & EUA & 157 & $40-79$ & $\begin{array}{l}\mathrm{AF} \text { e } \\
\mathrm{CS}\end{array}$ & $\leq 12$ meses & Médico & $\begin{array}{l}40 \text { - } 59 \text { anos, não fumantes, dislipidêmicos } \\
\text { e OB }\end{array}$ & Moderado \\
\hline Silagy et al. ${ }^{62}$ & ENG & 4941 & $35-64$ & ES & $\leq 12$ meses & $\begin{array}{l}\text { Médico e } \\
\text { Enfermeiro }\end{array}$ & Homens & Baixo \\
\hline Sinclair et al. ${ }^{63}$ & CAN & 1562 & $\geq 18$ & $\begin{array}{l}\mathrm{AF} \text { e } \\
\mathrm{AS}\end{array}$ & NR & Médico & Homens, 35 - 54 anos, $\uparrow \mathrm{n}^{\circ}$ DCNT & Baixo \\
\hline Siqueira et al. ${ }^{64}$ & $\mathrm{BR}$ & 8063 & $\geq 30$ & $\mathrm{AF}$ & Alguma vez & Médico & $\begin{array}{l}\text { Mulheres, avançar da idade, DCNT (DM e } \\
\text { e HAS), sedentários }\end{array}$ & Baixo \\
\hline $\begin{array}{l}\text { Tarasenko et } \\
\text { al. }{ }^{65}\end{array}$ & EUA & 42.275 & $\geq 18$ & $\mathrm{AF}$ & $\leq 12$ meses & Médico & Suficientemente ativos (com e sem câncer) & Baixo \\
\hline Wee et al. ${ }^{66}$ & EUA & 9711 & $\geq 18$ & $\mathrm{AF}$ & $\leq 12$ meses $^{b}$ & Médico & $\begin{array}{l}\geq 30 \text { anos, } \uparrow \text { níveis de AF, } \uparrow \text { IMC, DCNT } \\
\text { (DM e doença cardíaca) }\end{array}$ & Baixo \\
\hline $\begin{array}{l}\text { Weidinger et } \\
\text { al. }^{67}\end{array}$ & EUA & 1141 & $\geq 18$ & $\mathrm{AF}$ & $\leq 12$ meses & Médico & Mulheres, brancos & Baixo \\
\hline
\end{tabular}

a Estudo realizado com usuários e profissionais, classificado em ambas as categorias; b Informação não especificada na pergunta central da pesquisa, mas em outra parte do artigo. EUA = Estados Unidos da América; BR = Brasil; NZL = Nova Zelândia; GER = Alemanha; LIT $=$ Lituânia EUR = Europa; $\mathrm{ESP}=$ Espanha; $\mathrm{EST}=$ Estônia ENG = Inglaterra $; \mathrm{CA}=$ Canadá; $\mathrm{n}=$ amostra; $\mathrm{AF}=$ atividade física; $\mathrm{AS}=$ alimentação saudável; $\mathrm{ES}$ = estilo de vida; $\mathrm{CAM}$ = caminhada; $\mathrm{CS}$ = comportamento sedentário; $\mathrm{NR}$ = não reportado; HAS = hipertensão arterial sistêmica; $\mathrm{OB}=$ obesidade; $\mathrm{DCNT}=$ doenças crônicas não transmissíveis; IMC = índice de massa corporal; $\mathrm{MCU}=$ medicamentos de uso contínuo; $\mathrm{DM}=$ diabetes mellitus; $\uparrow$ = alto ou elevado; $\geq=$ maior ou igual; $\leq=$ menor ou igual. 
Prevalência de aconselhamento para atividade física referido pelos profissionais (\%)

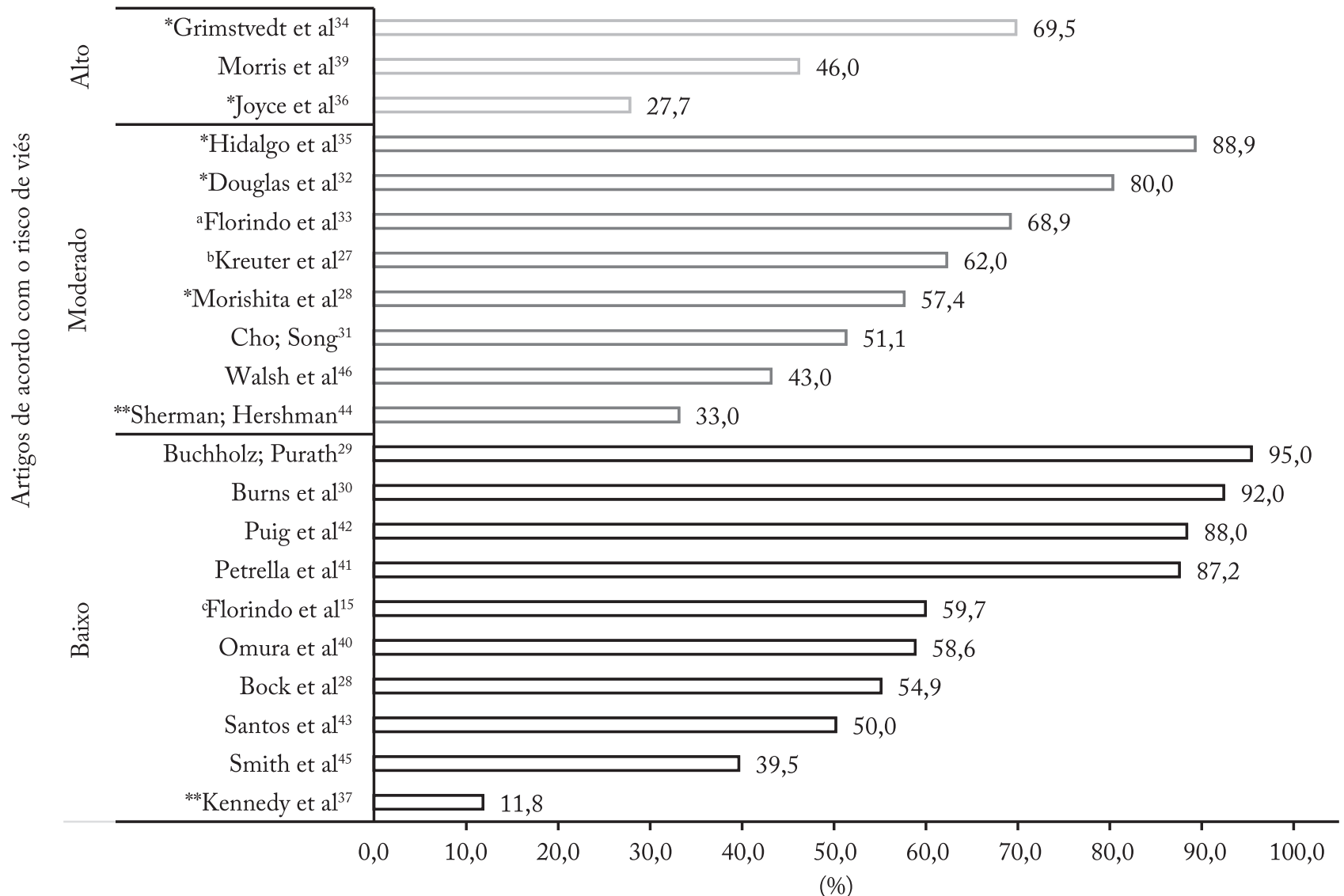

Figura 2 - Prevalência de aconselhamento para atividade física referido por profissionais de saúde da Atenção Básica à Saúde.

* Valor obtido pelo cálculo da média = entre profissionais ${ }^{31,33}$, entre morbidades ${ }^{35,38,45}$, e entre profissionais e morbidades ${ }^{34}$.**Aconselham cerca de $76 \%$ - 100\% dos pacientes. ${ }^{a} 2013$; ${ }^{\text {b }}$ estudo realizado com usuários e profissionais, classificado em ambas as categorias; ${ }^{\text {c }} 2015$.

risco de viés não produziram estimativas que diferem das gerais, assim parece que o risco de viés não foi significativo para as prevalências apresentadas.

A prevalência de aconselhamento referida pelos profissionais de saúde obteve média elevada, relativamente superior a referida pelos usuários. Em média, seis em cada dez profissionais relataram realizar aconselhamento para $\mathrm{AF}$ na $\mathrm{ABS}$. Esta revisão não se propôs a analisar a taxa de concordância entre os relatos de aconselhamento dos profissionais com usuários, até porque diversos fatores estão envolvidos, como a percepção do profissional, recordação do usuário ${ }^{68}$, e aspectos contextuais que distinguem o oferecer do receber aconselhamento; porém a diferença apresentada parece concordar com os resultados de um estudo ${ }^{27}$ que, ao pesquisar profissionais e usuários concomitantemente, apontou baixa taxa de concordância entre usuários e médicos.

Embora a maioria dos artigos com profissionais tenham apresentado prevalência acima de 50\%, houve uma certa variação entre os valores (entre 11,8\% a
95\%). Isso pode ser explicado pela pergunta central utilizada para identificar o aconselhamento, pois nem todos os estudos apresentaram a pergunta central, e dos que citaram, percebe-se variabilidade no uso das palavras "aconselha" e "recomenda" AF; na especificação da AF (30 minutos por dia de AF moderada ou AF como promoção de saúde ${ }^{30}$ ) e no tempo delimitado da realização do aconselhamento (pelo menos uma vez no ano $^{30}$ ou nos últimos seis meses ${ }^{43}$ ). Também houve variabilidade na opção das respostas, apresentadas em forma de escala sobre prontidão para aconselhar ${ }^{15,33}$, de porcentagem de pacientes aconselhados ${ }^{46}$, se aconselha AF diante de um caso clínico específico ${ }^{35}$, e para quais doenças recomenda a prática de exercícios físicos ${ }^{38}$. Além disso, a variação das prevalências de aconselhamento pode estar relacionada às diferentes características da prática assistencial de cada país ou profissional, como tempo, quantidade e local de atendimento, e o nível de vínculo que possui com os usuários.

A oferta de aconselhamento referida pelos profissionais de saúde é um fator importante, dada a cres- 
Prevalência de aconselhamento para atividade física recebido pelos usuários (\%)

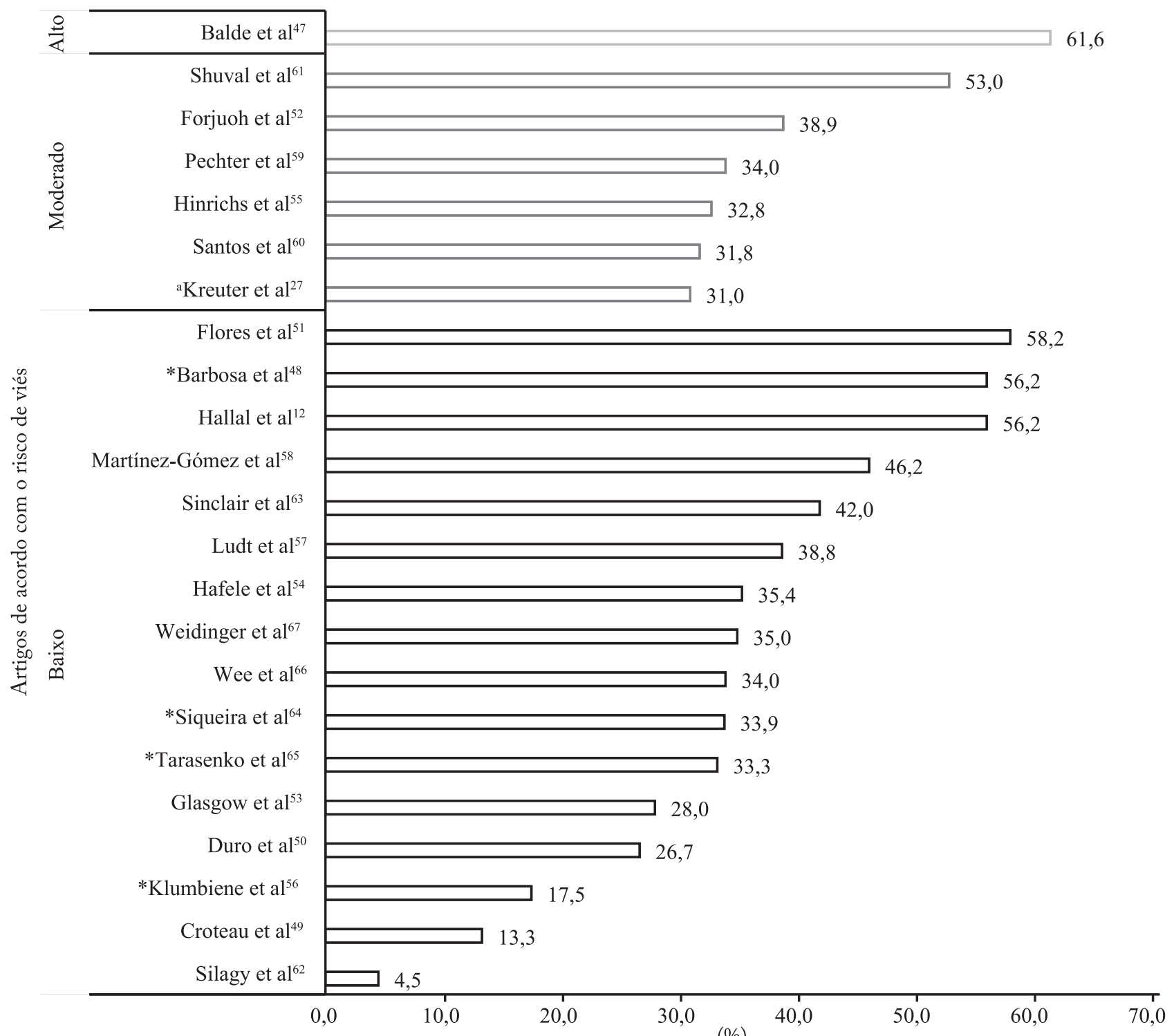

(\%)

Figura 3 - Prevalência de aconselhamento para atividade física recebido por usuários na Atenção Básica à Saúde.

*Valor obtido pelo cálculo da média = entre morbidades (diabéticos e hipertensos) ${ }^{48}$; entre idade ${ }^{64}$; entre nível de AF (inativos, insuficiente ativo e suficiente ativos ${ }^{65}$; entre $\operatorname{sexos}^{56}$. ${ }^{a}$ Estudo realizado com usuários e profissionais, classificado em ambas as categorias.

cente prevalência de inatividade física ${ }^{1,69,70}$. A variação apresentada na prevalência evidencia a importância da formação adequada no campo da educação em saúde e a necessidade de continuar fortalecendo os sistemas de saúde para abordar a promoção, prevenção e controle de doenças não transmissíveis e aconselhamento para AF no contexto da $\mathrm{ABS}^{13,60,71}$. Também é importante considerar as barreiras individuais e organizacionais que os profissionais de saúde enfrentam no cotidiano das práticas que os impede de aconselhar para $\mathrm{AF}$, como falta de tempo, de recursos específicos (protocolos de aconselhamento) e de treinamento ${ }^{22}$.
O médico foi o profissional de saúde mais entrevistado a respeito da realização de aconselhamento para AF. Esse predomínio foi percebido tanto nos estudos que investigaram apenas uma categoria profissional, quanto várias profissões. Como a maioria dos estudos investigaram o aconselhamento na perspectiva uniprofissional, não foi possível inferir a respeito de qual profissional de saúde aconselha mais para $\mathrm{AF}$, mas sim o profissional que foi mais investigado. Também, devido a quantidade mínima de estudos multiprofissionais optou-se por não identificar a prevalência de aconselhamento por cada profissional e sim calcular a média de forma geral. 
Os poucos estudos que avaliaram a realização de aconselhamento entre várias profissões encontraram que enfermeiro ${ }^{42}$, enfermeiro e $\mathrm{ACS}^{32}$, médico ${ }^{33}$, parteira $^{39}$ foram os profissionais que mais aconselhavam.

A centralidade do aconselhamento no profissional médico pode ser decorrente da configuração da ABS de cada país, mas precisa ser repensada. Segundo Hinrick et al. ${ }^{55}$; Tulloch et al. ${ }^{72}$ programas de aconselhamento precisam encontrar maneiras de incluir outros profissionais da área de saúde, além dos médicos, principalmente porque intervenções voltadas à mudança de comportamento requerem ações integradas de caráter multiprofissional e transdisciplinar. Neste aspecto, o Brasil apresenta-se como um modelo interessante devido a configuração da sua $\mathrm{ABS}$, que está pautada no trabalho multiprofissional em saúde por meio da Equipe de Saúde da Família (ESF) e do Núcleo Ampliado de Saúde da Família (NASF-AB) ${ }^{73}$. Essa configuração da ABS do país explica a tendência dos estudos brasileiros em não especificar o médico como único profissional a realizar aconselhamento para $\mathrm{AF}^{12,48,50,51,60} \mathrm{e}$ reconhece outros profissionais na promoção de saúde, como enfermeiro e agente comunitário de saúde ${ }^{15,35,43}$.

Embora considere a existência de equipe multiprofissional na $\mathrm{ABS}$, dos estudos que solicitaram para os usuários indicarem o profissional de saúde que mais aconselha para $\mathrm{AF}$, o médico aparece como o mais citado ${ }^{12,50,60}$ e os demais profissionais (nutricionista, profissional de educação física, agente comunitário de saúde) foram consideravelmente menos citados. A recente inserção desses profissionais no contexto da $\mathrm{ABS}$ e o predomínio do modelo biomédico nas práticas assistenciais ${ }^{74}$ pode ter influenciado a percepção dos usuários quanto ao reconhecimento/visibilidade. Isso reforça a necessidade de ações de educação permanente a fim de instrumentalizar todos profissionais de saúde a desenvolverem aconselhamento sobre estilos de vida saudáveis de modo interdisciplinar e com responsabilidade compartilhada ${ }^{72,73,75}$.

Aproximadamente, três em cada dez usuários relataram ter recebido aconselhamento para $\mathrm{AF}$ na $\mathrm{ABS}$. De modo geral, a prevalência de aconselhamento referido pelos usuários foi abaixo de $40 \%$. A baixa prevalência pode estar relacionada a diversos fatores como, subnotificação, falta de homogeneidade dos participantes, barreiras para aconselhamento entre os diferentes profissionais de saúde e diferentes configurações da ABS. Há de se considerar também que houve algumas variações na questão central das pesquisas: tempo de recordação do aconselhamento; profissional referen- ciado na pergunta e maneira como foi apresentada a prevalência, ora dividida por sexo, faixa etária, categoria profissional ou características do grupo.

As prevalências mais elevadas de aconselhamento para AF foram encontradas nos estudos de Hallal et al. ${ }^{12}$, Balde et al. ${ }^{47}$, Barbosa et al. ${ }^{48}$, Flores et al. ${ }^{51} \mathrm{e}$ Shuval et al. ${ }^{61}$, com valores de aconselhamento acima de 50\%. Tendo em vista que o aconselhamento é uma técnica que deve ser incorporada na $\mathrm{ABS}^{13}$, a baixa prevalência reportada pelos usuários adverte para o desenvolvimento de métodos para utilização do aconselhamento como ferramenta de promoção da $\mathrm{AF}^{19,75}$.

Os artigos incluídos mostram que usuários que mais recebem aconselhamento para $\mathrm{AF}$ apresentam excesso de peso corporal, obesidade e doenças crônicas não transmissíveis. $\mathrm{O}$ aconselhamento para $\mathrm{AF}$ realizado na $\mathrm{ABS}$ representa uma oportunidade para abordar a temática atividade física juntamente com outros comportamentos preventivos ligados à saúde, já que profissionais de saúde podem influenciar positivamente na mudança de comportamento e adoção de hábitos saudáveis ${ }^{76-78}$. Nesse sentido, o recebimento de aconselhamento para $\mathrm{AF}$ por pessoas com saúde comprometida é bom resultado, pois demonstra que os profissionais de saúde, de certo modo, consideram os benefícios da prática de $\mathrm{AF}$ no controle das morbidades ${ }^{54}$. Porém, é igualmente importante assegurar que o aconselhamento atenda não apenas as necessidades imediatas de saúde ou de controle de doenças, mas também a pessoas aparentemente saudáveis ${ }^{79}$.

Dispensar o aconselhamento para $\mathrm{AF}$ a pessoas sem diagnóstico de doenças crônicas pode representar importantes oportunidades perdidas, uma vez que a ABS se caracteriza não só por desenvolver ações de prevenção, mas de promoção e manutenção da saúde ${ }^{7,66}$. Assim, fomentar aconselhamento para AF sob a ótica da promoção da saúde constitui um grande desafio, visto que a maioria das práticas de saúde da $\mathrm{ABS}$ se organizam em torno de conceitos de doenças, assim como também as diretrizes e recomendações de $\mathrm{AF}^{80}$.

Ao nosso conhecimento, essa é a primeira revisão sistemática a respeito da prevalência de aconselhamento para AF no contexto da ABS. Esta revisão identificou estudos realizados tanto com profissionais como com usuários, reportando as prevalências de aconselhamento sob as perspectivas do oferecimento e do recebimento. Como limitação do estudo, não foi realizada a busca na literatura cinzenta (publicações não indexadas), portanto, é possível que algum estudo não tenha sido identificado. No entanto, foram pesquisadas sete 
bases de periódicos, além da leitura nas referências dos artigos selecionados e consulta a experts, o que aumenta a sensibilidade na busca de artigos.

Pensando em implicações práticas, a promoção da AF por meio do aconselhamento pode ser maximizada se outros profissionais de saúde, além do médico, participarem do processo. Para isso, o incentivo ao aconselhamento de caráter multiprofissional que busque $o$ diálogo com outros setores, como fundações de esportes e conselhos comunitários, poderá fortalecer a adesão a prática de AF. Também, é necessário testar instrumentos aplicáveis para aumentar a prevalência de aconselhamento na $\mathrm{ABS}$, bem como realizar intervenções que tenham como foco a promoção da $\mathrm{AF}$ em indivíduos aparentemente saudáveis e como forma de tratamento a indivíduos com doenças crônicas não transmissíveis. Adicionalmente, é importante que as políticas públicas de aconselhamento para AF sejam incentivadas e fortalecidas com a implementação de esquemas de referência, protocolos e suportes estruturais (alertas, roteiros de aconselhamento e recursos de rastreamento), visando tornar o aconselhamento para $\mathrm{AF}$ uma prática cotidiana dos serviços de ABS.

Conclui-se que a prevalência de aconselhamento para AF referida pelos profissionais de saúde foi cerca de metade enquanto que a referida pelos usuários foi abaixo. $\mathrm{O}$ aconselhamento para $\mathrm{AF}$ ainda é incipiente na ABS, especialmente pela baixa percepção de aconselhamento recebido pelos usuários. A análise das prevalências, onde em média, seis em cada dez profissionais relataram aconselhar para $\mathrm{AF}$ na $\mathrm{ABS}$, e três em cada dez usuários relataram ter recebido aconselhamento, destaca a importância de fomentar a prática de aconselhamento nos serviços de ABS, visto a crescente prevalência de inatividade física; além do desenvolvimento de estudos que consigam responder ao máximo as características que permeiam o aconselhamento: qual profissional aconselha AF, como, com qual instrumento e em quanto tempo é realizado, o que exatamente é aconselhado e detalhes da estrutura da ABS.

\section{Conflito de interesse}

Os autores declaram não haver conflito de interesses.

\section{Contribuição dos autores}

Moraes SQ, foi responsável pela seleção dos artigos, coleta; análise e interpretação dos resultados e redação do manuscrito. Souza $\mathrm{JH}$, foi responsável pela pesquisa bibliográfica, avaliação, seleção dos artigos, coleta e análise dos dados. Araújo PAB, foi respon- sável pela pesquisa bibliográfica, avaliação, seleção dos artigos, coleta e análise dos dados e redação do manuscrito. Rech CR, participou da concepção inicial do estudo, avaliação, análise de dados e contribuiu na redação e revisão crítica do manuscrito.

\section{Agradecimentos}

Os autores gostariam de agradecer ao Grupo de Estudos e Pesquisa em Ambiente Urbano \& Saúde e aos integrantes do Projeto Aconselha SUS.

\section{Referências}

1. Guthold R, Stevens GA, Riley LM, Bull FC. Worldwide trends in insuficiente physical activity from 2001 to 2016: a pooled analysis of 358 population-based surveys with 1.9 million participants. Lancet Glob Health. 2018;6(10):1077-86.

2. Matthews CE, Cohen SS, Fowke JH, Han X, Xiao Q, Buchowski MS, et al. Physical activity, sedentary behavior, and cause-specific mortality in black and white adults in the Southern Community Cohort Study. Am J Epidemiol. 2014;180(4):394-405.

3. Lee IM, Shiroma EJ, Lobelo F, Puska P, Blair SN, Katzmarzyk PT, et al. Effect of physical inactivity on major noncommunicable diseases worlwide: na analysis of burden of disease and life expectancy. Lancet. 2012;380(9838):219-29.

4. Hallal PC, Andersen LB, Bull FC. Guthold R, Haskell W, Ekelund U, et al. Global physical activity levels: surveillance progress, pitfalls, and prospects. Lancet. 2012;380(9838):247-57.

5. World Health Organization (WHO). Global recommendations on physical activity for health. Geneva: WHO; 2010.

6. Sallis JF, Bull F, Guthold R, Heath GW, Inoue S, Kelly P, et al. Progress in physical activity over the olympic quadrennium. Lancet. 2016;388(10051):1325-36.

7. Grossman DC, Bibbins-Domingo K, Curry SJ, Barry MJ, Davidson KW, Doubeni CA, et al. Behavioral counseling to promote a healthful diet and physical activity for cardiovascular disease prevention in adults without cardiovascular risk factors: US Preventive Services Task Force Recommendation Statement. JAMA. 2017;318(2);167-74.

8. Orrow G, Kinmonth AL, Sanderson S, Sutton S. Republished research: effectiveness of physical activity promotion based in primary care: systematic review and meta-analysis of randomised controlled trials. Br J Sports Med. 2013;47(1):e1389.

9. Williams NH. "The wise, for cure, on exercise depend": physical activity interventions in primary care in Wales. Br J Sports Med. 2009;43(2):106-8.

10. Vuori IM, Lavie CJ, Blair SN. Physical activity promotion in the health care system. Mayo Clin Proc. 2013;88(12):1446-61.

11. Gagliardi AR, Abdallah F, Faulkner G, Ciliska D, Hicks A. Factors contributing to the effectiveness of physical activity counselling in primary care: a realist systematic review. Patient Educ Couns. 2015;98(4):412-9.

12. Hallal PC, Machado PT, Del Duca GF, Silva IC, Amorim TC, Borges TT, et al. Physical activity advice: short report from a population-based study in Brazil. J Phys Act Health. 2010;7(3):352-54.

13. Brasil. Ministério da Saúde (MS). Secretaria de Vigilância em Saúde. Secretaria de Atenção à Saúde. Política Nacional de Promoção da Saúde. Brasília: MS; 2014. [citado 2018 jan 20]. Disponível em: http://bvsms.saude.gov.br/bvs/ saudelegis/gm/2014/prt2446_11_11_2014.html. 
14. Brasil. Ministério da Saúde (MS). Secretaria de Atenção à Saúde. Departamento de Atenção Básica. Política Nacional de Atenção Básica. Brasília: MS; 2012. [citado 2018 jan 20]. Disponível em: http://189.28.128.100/dab/docs/ publicacoes/geral/pnab.pdf.

15. Florindo AA, Brownson RC, Mielke GI, Gomes GAO, Parra DC, Siqueira FV, et al. Association of knowledge, preventive counseling and personal health behaviors on physical activity and consumption of fruits or vegetables in community health workers. BMC Public Health. 2015;15(1):344.

16. Sanchez A, Bully P, Martinez C, Grandes G. Effectiveness of physical activity promotion intervention in primary care: $\mathrm{a}$ review of review. Prev Med. 2015;76(Supl.):56-67.

17. Jacobson DM, Strohecker L, Compton MT, Katz DL. Physical activity counseling in the adult primary care setting: position statement of the American College of Preventive Medicine. Am J Prev Med. 2005;29(2):158-62.

18. EU Working Group “Sport \& Health”. EU Physical Activity Guidelines. Recommended Policy Actions in Support of Health-Enhancing Physical Activity. Brussels: EU; 2008.

19. Short CE, Hayman M, Rebar AL, Gunn KM, De Cocker K, Duncan MJ, et al. Physical activity recommendations from general practitioners in Australia. Results from a national survey. Aust N Z J Public Health. 2016;40(1):83-90.

20. Lawlor DA, Hanratty B. The effect of physical activity advice given in routine primary care consultations: a systematic review. J Public Health Med. 2001;23(3):219-26.

21. Petrella RJ, Lattanzio CN. Does counseling help patients get active? Systematic review of the literature. Can Fam Physician. 2002;48:72-80.

22. Hébert ET, Caughy MO, Shuval K. Primary care providers' perceptions of physical activity counselling in a clinical setting: a systematic review. Br J Sports Med. 2012;46(9):625-31.

23. Dacey ML, Kennedy MA, Polak R, Phillips EM. Physical activity counseling in medical school education: a systematic review. Med Educ Online. 2014;19(1):24325.

24. Moher D, Liberati A, Tetzlaff J, Altman DG. PRISMA Group. Preferred reporting items for systematic reviews and meta-analyses: the PRISMA statement. PLoS Med. 2009;6(7):e1000097.

25. Munn Z, Moola S, Lisy K, Riitano D, Tufanaru C. Methodological guidance for systematic reviews of observational epidemiological studies reporting prevalence and cumulative incidence data. Int J Evid Based Health. 2015;13(3):147-53.

26. Aromataris E, Munn Z. Joanna Briggs Institute Reviewer's Manual. The Joanna Briggs Institute. 2017. Disponível em: https://reviewersmanual.joannabriggs.org/

27. Kreuter MW, Scharff DP, Brennan LK, Lukwago SN. Physician recommendations for diet and physical activity: which patients get advised to change?. Prev Med. 1997;26(6):825-33.

28. Bock C, Diehm C, Schneider S. Physical activity promotion in primary health care: results from a German physician survey. Eur J Gen Pract. 2012;18(2):86-91.

29. Buchholz SW, Purath J. Physical activity and physical fitness counseling patterns of adult nurse practitioners. J Am Acad Nurse Pract. 2007;19(2):86-92.

30. Burns KJ, Camaione DN, Chatterton CT. Prescription of physical activity by adult nurse practitioners: a national survey. Nurs Outlook. 2000;48(1);28-33.

31. Cho HJ, Sunwoo S, Song YM. Attitudes and reported practices of Korean primary care physicians for health promotion. Journal Korean Med Sci. 2003;18(6):783-90.
32. Douglas F, Torrance N, Van Teijlingen E, Meloni S, Kerr A. Primary care staff s views and experiences related to routinely advising patients about physical activity. A questionnaire survey. BMC Public Health. 2006;6:138.

33. Florindo AA, Mielke GI, Gomes GAO, Ramos LR, Bracco MM, Parra DC, et al. Physical activity counseling in primary health care in Brazil: a national study on prevalence and associated factors. BMC Public Health 2013;13(1):794.

34. Grimstvedt ME, Der Ananian C, Keller C, Woolf K, Sebren A, Ainsworth B. Nurse practitioner and physician assistant physical activity counseling knowledge, confidence and practices. Prev Med. 2012;54(5);306-8.

35. Hidalgo KD, Mielke GI, Parra DC, Lobelo F, Simôes EJ, Gomes GO, et al. Health promoting practices and personal lifestyle behaviors of Brazillian health professionals. BMC Public Health. 2016;16(1):1114.

36. Joyce CL, O'Tuathaigh CM. Increased training of general practitioners in Ireland may increase the frequency of exercise counselling in patients with chronic illness: a cross-sectional study. Eur J Gen Pract. 2014;20(4):314-9.

37. Kennedy MF, Meeuwisse WH. Exercise counselling by family physicians in Canada. Prev Med. 2003;37(3):226-32.

38. Morishita Y, Miki A, Okada M, Tsuboi S, Ishibashi K, Ando $\mathrm{Y}$, et al. Exercise counseling of primary care physicians in metabolic syndrome and cardiovascular diseases is associated with their specialty and exercise habits. Int J Gen Med. 2014;7:277-83.

39. Morris J, Nikolopoulos H, Berry T, Jain V, Vallis M, PiccininiVallis $\mathrm{H}$, et al. Healthcare providers' gestational weight gain counselling practises and the influence of knowledge and attitudes: a cross-sectional mixed methods study. BMJ Open. 2017;7(11):018527.

40. Omura JD, Bellissimo MP, Watson KB, Loustalot F, Fulton JE, Carlson SA. Primary care providers' physical activity counseling and referral practices and barriers for cardiovascular disease prevention. Prev Med. 2018;108:115-22.

41. Petrella RJ, Lattanzio CN, Overend TJ. Physical activity counseling and prescription among canadian primary care physicians. Arch Intern Med. 2007; 167(16):1774-81.

42. Puig RA, McKenna J, Riddoch C. Attitudes and practices of physicians and nurses regarding physical activity promotion in the Catalan primary health-care system. Eur J Public Health. 2005;15(6):569-75.

43. Santos TI, Guerra PH, Andrade DR, Florindo AA. Práticas pessoais e profissionais de promoção da atividade física em agentes comunitários de saúde. Rev Bras Ativ Fís Saúde. 2015;20(2);165-73.

44. Sherman SE, Hershman WY. Exercise counseling. How do general internists do? J Gen Intern Med. 1993;8(5);243-48.

45. Smith AW, Borowski LA, Liu B, Galuska DA, Signore C, Klabunde C, et al. U.S. primary care physicians' diet, physical activity, and weight-related care of adult patients. Am J Prev Med. 2011;41(1):33-42.

46. Walsh JM, Swangard DM, Davis T, McPhee SJ. Exercise counseling by primary care physicians in the era of managed care. Am J Prev Med. 1999;16(4):307-13.

47. Balde A, Figueras J, Hawking DA, Miller JR. Physician advice to the elderly about physical activity. J Aging and Phys Act. 2003;11(1):90-7.

48. Barbosa JMV, Souza WV, Ferreira RWM, Carvalho EMF, Cesse EAP, Fontbonne A. Correlates of physical activity counseling by health providers to patients with diabetes and hypertension attended by the Family Health Strategy in the state of Pernambuco, Brazil. Prim Care diabetes. 2017;11(4):327-36.

49. Croteau K, Schofield G. McLean G. Physical activity advice in the primary care setting: results of a population study in New Zealand. Aust N Z J Public Health. 2006;30(3);262-67. 
50. Duro SM, Tomasi E, Siqueira FV, Silveira DS, Thumé E, Facchini LA. Adult physical activity counseling by health professionals in Brazil: a national urban population survey. J Phys Act Health. 2015;12(8):1177-83.

51. Flores TR, Gomes AP, Soares ALG, Nunes BP, Assunção $\mathrm{MC}$, Gonçalves $\mathrm{H}$, et al. Aconselhamento por profissionais de saúde e comportamentos saudáveis entre idosos: estudo de base populacional em Pelotas, sul do Brasil, 2014. Epidemiol Serv Saude. 2018;27(1):e201720112.

52. Forjuoh SN, Lee C, Won J, Towne SD, Wang S, Ory MG. Correlates of receiving a recommendation for more physical activity from a primary care provider. Am J Prev Med. 2017;52(2);207-14.

53. Glasgow RE, Eakin EG, Fisher EB, Bacak SJ, Brownson RC. Physician advice and support for physical activity: results from a national survey. Am J Prev Med. 2001;21(3):189-96.

54. Häfele V, Siqueira F. Aconselhamento para atividade física e mudança de comportamento em Unidades Básicas de Saúde. Rev Bras Ativ Fís Saúde. 2017;21(6):581-92.

55. Hinrichs T, Moschny A, Klaassen-Mielke R, Trampisch U, Thiem U, Platen P. General practitioner advice on physical activity: analyses in a cohort of older primary health care patients (getABI). BMC Fam Pract. 2011;12(1):26.

56. Klumbiene J, Petkeviciene J, Vaisvalavicius V, Miseviciene I. Advising overweight persons about diet and physical activity in primary health care: Lithuanian health behaviour monitoring study. BMC Public Health. 2006;6(1):30.

57. Ludt S,PetekD, Laux G, Van Lieshout J, Campbell SM, Kunzi $\mathrm{B}$, et al. Recording of risk-factors and lifestyle counselling in patients at high risk for cardiovascular diseases in European primary care. Eur J Prev Cardiol. 2012;19(2):258-66.

58. Martínez-Gomez D, León-Muñoz LM, Guallar-Castillón P, López-Garcia E, Aguilera MT, Banegas JR, et al. Reach and equity of primary care-based counseling to promote walking among the adult population of Spain. J Sci Med Sport. 2013;16(6):532-8.

59. Pechter U, Suija K, Kordemets T, Kalda R, Maaroos HI. Physical activity and exercise counselling: a cross-sectional study of Family practice patients in Estonia. Qual Prim Care. 2012;20(5):355-63.

60. Santos RP, Horta PM, Souza CS, Santos CA, Oliveira HBS, Almeida LMR, et al. Aconselhamento sobre alimentação e atividade física: prática e adesão de usuários da atenção primária. Rev Gaúcha de Enferm. 2012;33(4):14-21.

61. Shuval K, DiPietro L, Skinner CS, Barlow CE, Morrow J, Goldsteen R, et al. 'Sedentary behaviour counselling': the next step in lifestyle counselling in primary care; pilot findings from the Rapid Assessment Disuse Index (RADI) study. Br J Sports Med. 2014;48(19):1451-5.

62. Silagy C, Muir J, Coulter A, Thorogood M, Yudkin P, Roe L. Lifestyle advice in general practice: rates recalled by patients. BMJ. 1992;305(6858):871-74.

63. Sinclair J, Lawson B, Burge F. Which patients receive on diet and exercise? Do certain characteristics affect whether they receive such advice?. Can Fam Physician. 2008;54(3):404-12.

64. Siqueira FV, Nahas MV, Facchini LA, Silveira DS, Piccini RX, Tomasi E, et al. Aconselhamento para a prática de atividade física como estratégia de educação à saúde. Cad. Saude Publica. 2009;25(1):203-13.
65. Tarasenko YN, Miller EA, Chen C, Schoenberg NE. Physical activity levels and counseling by health care providers in cancer survivors. Prev Med. 2017;99:211-17.

66. Wee CC, McCarthy EP, Davis RB, Phillips RS. Physician counseling about exercise. JAMA. 1999;282(16):1583-8.

67. Weidinger KA, Lovegreen SL, Elliot MB, Hagood L, HaireJoshu D, McGill JB, et al. How to make exercise counseling more effective: lessons from rural America. J Fam Practic. 2008;57(6):394-402.

68. Bardach SH, Schoenberg NE, Howell BM. Older patient's recall of lifestyle discussions in primary care.J Appl Gerontol. 2017;36(4):386-400.

69. Brasil. Ministério da Saúde.Vigitel Brasil 2017: vigilância de fatores de risco e proteção para doenças crônicas por inquérito telefônico: estimativas sobre frequência e distribuição sociodemográficas de fatores de risco e proteção para doenças crônicas nas capitais dos 26 estados brasileiros e no Distrito Federal em 2017. Brasília: MS; 2018.

70. World Health Organization (WHO). Global status report on noncommunicable diseases. Geneva: WHO; 2014.

71. World Health Organization (WHO). Global action plan for the prevention and control of noncommunicable diseases 2013-2020. Geneva: WHO; 2013.

72. Tulloch H, Fortier M, Hogg W. Physical activity counseling in primary care: who has and who should be counseling? Patient Educ Couns. 2006;64(1-3):6-20.

73. Brasil. Ministério da Saúde (MS). Cadernos de Atenção Básica. Núcleo de Apoio à Saúde da Família. Brasília: MS; 2014.

74. Fertonani HP, Pires DEP, Biff D, Scherer MDA. The health care model: concepts and challenges for primary health care in Brazil. Cienc Saúde Coletiva. 2015;20(6):1869-78.

75. Aittasalo M, Kukkonen-Harjula K, Toropainen E, Rinne M, Tokola K, Vasankari, T. Developing physical activity counseling in primary care through participatory action approach. BMC Fam Prac. 2016;17(141):1-15.

76. Berry LL, Flynn AG, Seiders K, Haws KL, Quach SQ. Physician counseling of overweight patients about preventive health behaviors. Am J Prev Med. 2014;46(3):297-302.

77. Jennings A, Barnes S, Okereke U, Welch A. Successful weight management and health behaviour change using a health trainer model. Perspect Public Health. 2013;133(4);221-6.

78. Hung OY, Keenan NL, Fang J. Physicians' health habits are associated with lifestyle counseling for hypertensive patients. Am J Hypertens. 2013;26(2):201-8.

79. Lopes ACS, Toledo MTT, Câmara AMCS, Menzel HJK, Santos, LC. Condições de saúde e aconselhamento sobre alimentação e atividade física na Atenção Primária à Saúde de Belo Horizonte-MG. Epidemiol Serv Saúde. 2014;23(3);475-86.

80. Czeresnia D. O conceito de saúde e a diferença entre promoção e prevenção. In: Czeresnia D, Freitas CM (Org.). Promoção da Saúde: conceitos, práticas e reflexões. Rio de Janeiro: Fiocruz; 2003. p. 39-53.

Recebido: 26/02/2019

Aprovado: 06/08/2019 